\title{
Stability Analysis Method of AC / DC UHV Power System Based on Risk Assessment
}

\author{
Mingxin Xu ${ }^{1, a}$, Long Zhao ${ }^{1, a}$, Rishang Long ${ }^{2, a}$, Jianhua Zhang ${ }^{2}$, Yang Liu ${ }^{2}$, \\ $\mathrm{Hao} \mathrm{Hu}{ }^{2}$, Tao Tan ${ }^{2}$, Yuan Meng ${ }^{2}$, Wenwen $\mathrm{Ma}^{2}$ \\ ${ }^{1}$ Inner Mongolia Eastern Electric Power Co., Ltd. Economic and Technical Research Institute, Inner \\ Mongolia, China, 010010 \\ ${ }^{2}$ State Key Laboratory of new energy power system, North China Electric Power University, Beijing, \\ China, 102206 \\ alrs18810667721@163.com
}

Keywords: AC / DC UHV power system, risk assessment, stability analysis

Abstract. The Ultra High Voltage power system plays an important role in alleviating the energy crisis. However, due to the high voltage level and complex operation, it will bring disaster to the system once the power grid fails, causing cascading failures and large area power outages. In this paper, we study the stability analysis method of AC/DC Ultra High Voltage power system. Based on setting fault scenarios and BPA simulation, we introduce risk assessment model and set up a series of indexes, including the low voltage risk, the unit angle difference risk and frequency instability risk, to assess the system stability. Compared with the traditional reliability analysis method, this paper combines the probability of the accident and its consequences, and links the risk and benefits, which can quantitatively reflects the safety index of the system. Therefore, it is more applicable in stability analysis for complex AC/DC Ultra High Voltage power system.

\section{Introduction}

Due to uneven distribution of the energy center and the load center in China, the construction of long distance, large capacity, low energy loss Ultra High Voltage (UHV) transmission system, is one way to achieve optimal allocation of energy and the coordinated development of economy and society ${ }^{[1-2]}$. However, with the continuous development of the power grid, the voltage level is gradually increased and the power system is more complex, showing a strong dynamic and nonlinear characteristics. This also leads to more complex in the power grid fault form, fault propagation and fault consequences, which increase difficulty to analysis the safety and stability of the system.

At present, the stability evaluation of power system is mainly dependent on the reliability theory. Reliability evaluation has penetrated into the power system planning, design, operation process, in order to achieve the comprehensive efficiency of the power system, and play a positive role in the safe and reliable operation of power system. Reliability analysis mainly includes analytical method and simulation method. References[3-4] presents the combination of collapse point method and extension method for the voltage stability analysis of AC / DC system, but not for UHV system; In [5], a new method for the identification of the voltage instability mode and power angle instability mode is proposed, but not fully considers fault scenarios. The above references are analytical method. When the system is too large, it is difficult to simulate multiple faults. Hierarchical sampling method is proposed in [6-7], which is based on the layered reliability model and combines the same failure modes into equivalent components, reducing the amount of computation; References [8-9] combines power system probabilistic sufficiency and probabilistic stability assessment to find some occurrence probability but small fault status of serious consequences, and BPA software is introduced in the calculation process, so as to shorten the computation time.

However, with the huge scale of system, various voltage levels and mixed operation of DC system, various uncertain factors begin to influence power system, causing that the traditional reliability assessment methods can not fully meet the need of power system security analysis. The traditional reliability assessment is mainly aimed at the probability of the accident, but usually do not consider 
the consequences of the accident. The shortcomings of its exposure is particularly evident while making the assessment of a number of accidents which have a small probability of occurrence, but the consequences is serious.

In 1997, the concept of operation risk assessment of power system was first proposed. The risk theory gives attention to both the probability of accident and the consequences, which makes the results more reasonable and instructive. At present, the risk assessment theory is mainly reflected in three aspects ${ }^{[10-13]}$ : the risk assessment of the market environment and economic factors, the risk assessment based on complex network theory, the online assessment of power system risk.

In this paper, the risk assessment theory is used to analyze the power system after cascading failures. Based on BPA simulation and the risk index, we evaluate the stability of the system. In the case study, the two planning scheme of SANHUA gird is calculated, which proves that the method is scientific and advantageous.

\section{Risk Assessment Theory}

\section{Basic Concept.}

Power system risk assessment is comprehensive measurement of the possibility and severity. Its formula is:

$$
R\left(X_{t, f}\right)=\sum_{i}^{n} P_{r}\left(E_{i}\right)\left(\sum_{j}^{m} P_{r}\left(X_{t, j} \mid X_{t, f}\right) S_{e v}\left(E_{i}, X_{t, j}\right)\right)
$$

In which $X_{t, f}$ is the predictive state at time $t, E_{i}$ is the $i$ th accident, $P_{r}\left(E_{i}\right)$ is the probability of the $i$ th accident; $X_{t, j}$ is the $j$ th possible system state at time $t ; P_{r}\left(X_{t, j} \mid X_{t, f}\right)$ is the probability of $X_{t, j} ; S_{e v}\left(E_{i}, X_{t, j}\right)$ represents the severity, such as overload, low voltage, loss of load, etc.

Risk assessment generally consists of four steps: (1)Determine component outage model; (2)Select the system failure state and calculate the probability of the system; (3)Evaluate the consequences of the selected state; (4)Calculate risk index. How to establish the failure mode and failure state of the system is the key point of the evaluation method. Generally, according to the N-1 stability calculation results and historical natural conditions, we select the important (such as the contact line), the voltage is weak, the disaster accident prone line as the trigger accident.

\section{Severity Function.}

After the occurrence of the power grid, there will appear some special state, including the bus voltage drop, line overload, load rejection, voltage collapse, etc. Here are three severity function used in this paper:

(1) Line low voltage severity function

Figure 1 shows the two kinds of severity function. Discrete function is defined as: when the bus voltage value is lower than the defined voltage threshold value 0.95 , the function value is 1 , otherwise the function value is 0 . Continuous function is defined as: when the bus voltage is 0.95 , the function values is 1 , and when the voltage level is further decreased, the function value increases linearly. It overcomes the shortcomings of the discrete function, and the size of the function value can reflect the level of the voltage level.

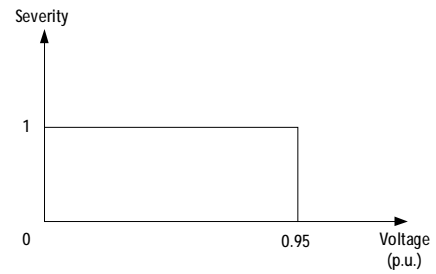

(a) Discrete function

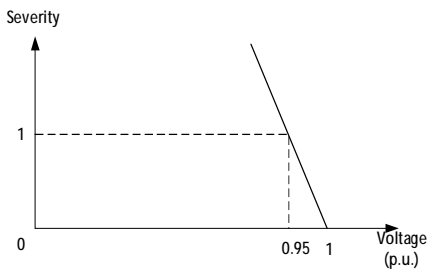

(b) Continuous function

Figure 1. Low voltage severity function

When the discrete function is used, the formula is: 


$$
S\left(U_{i j}\right)= \begin{cases}1 & U_{i j}<0.95 \\ 0 & U_{i j} \geq 0.95\end{cases}
$$

Where $U_{i j}$ is the voltage for bus $j$ under the $i$ th accident. $S\left(U_{i j}\right)$ is the low voltage severity function.

When the continuous function is used, the formula is:

$$
S\left(U_{i j}\right)=-20 U_{i j}+20
$$

In this paper, the continuous function is used, and the low voltage severity function is defined as:

$$
S\left(U_{i j}\right)=\omega_{u 1} \bar{S}\left(U_{i j}\right)+\omega_{u 2} S_{\max }\left(U_{i j}\right)
$$

Where $\omega_{u 1}$ and $\omega_{u 2}$ is the weight coefficient of severity, we take $\omega_{u 1}=\omega_{u 2}=0.5 . \bar{S}\left(U_{i j}\right)$ is the average voltage severity, $S_{\max }\left(U_{i j}\right)$ is the maximum voltage severity.

(2) The unit angle difference severe function

Similar to the low voltage severity function, the unit angle difference severe function is determined by the unit operation condition, which can be divided into 2 types: discrete type and continuous type, as shown in Figure 2:

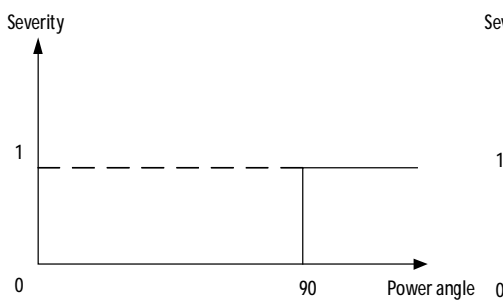

(a) Discrete function

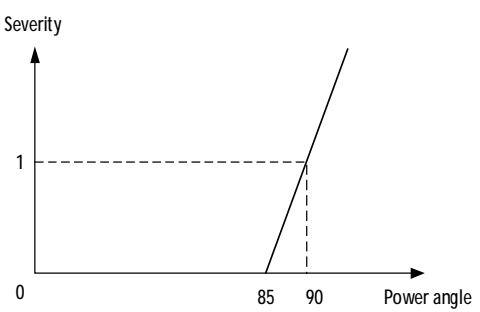

(b) Continuous function

Figure 2. The unit angle difference severity function

When the discrete function is used, the formula is:

$$
S\left(\delta_{i j}\right)= \begin{cases}1 & \delta_{i j}>90^{\circ} \\ 0 & \delta_{i j} \leq 90^{\circ}\end{cases}
$$

Where $\delta_{i j}$ is the unit angle for bus $j$ under the $i$ th accident. $S\left(\delta_{i j}\right)$ is the unit angle difference severe function.

When the continuous function is used, the formula is:

$$
S\left(\delta_{i j}\right)=0.20 \delta_{i j}-17
$$

In this paper, the continuous function is used, and the unit angle difference severity function is defined as:

$$
S\left(\delta_{i j}\right)=\omega_{\delta 1} \bar{S}\left(\delta_{i j}\right)+\omega_{\delta 2} S_{\max }\left(\delta_{i j}\right)
$$

Where $\omega_{\delta 1}$ and $\omega_{\delta 2}$ is the weight coefficient of severity, we take $\omega_{u 1}=\omega_{u 2}=0.5 . \bar{S}\left(\delta_{i j}\right)$ is the average unit angle difference severity, $S_{\max }\left(\delta_{i j}\right)$ is the maximum unit angle difference severity.

(3) The abnormal system frequency severity function

When a serious fault occurs in the power grid, it may lead to frequency instability. The system frequency severity can be expressed as the form below: 

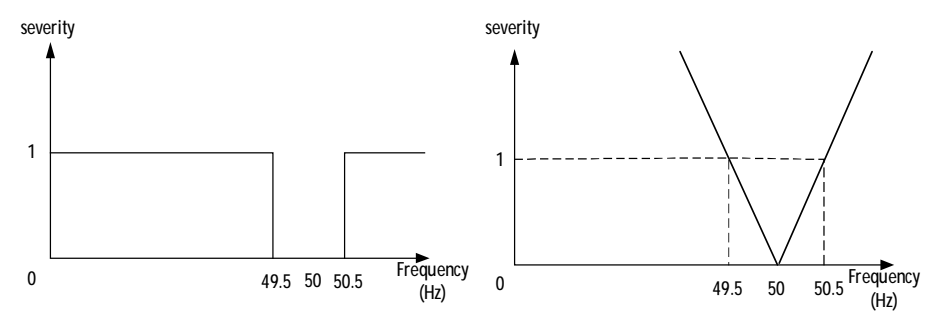

(a) Discrete function

(b) Continuous function

Figure 3. The system frequency severity function

When the discrete function is used, the formula is:

$$
S(f)= \begin{cases}1 & f>50.5 \text { orf }<49.5 \\ 0 & 49.5 \leq f \leq 50.5\end{cases}
$$

Where $f$ is the frequency,

When the continuous function is used, the formula is:

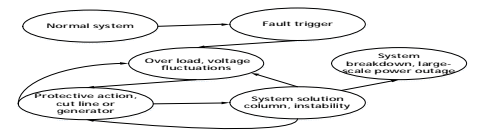

In this paper, the continuous function is used.

\section{Risk Assessment Model Based on Cascading Failure of Power System}

Usually, serious natural disasters, such as earthquake, typhoon and ice disaster, can cause a cascading failure, therefore, we should first clear chain reaction of the various stages, then calculate the risk value at all stages and the final cascading failure risk value. In this paper, the power system state is simulated by BPA program, and the development process of cascading failure is simulated. The general development process of cascading failures is as follows:

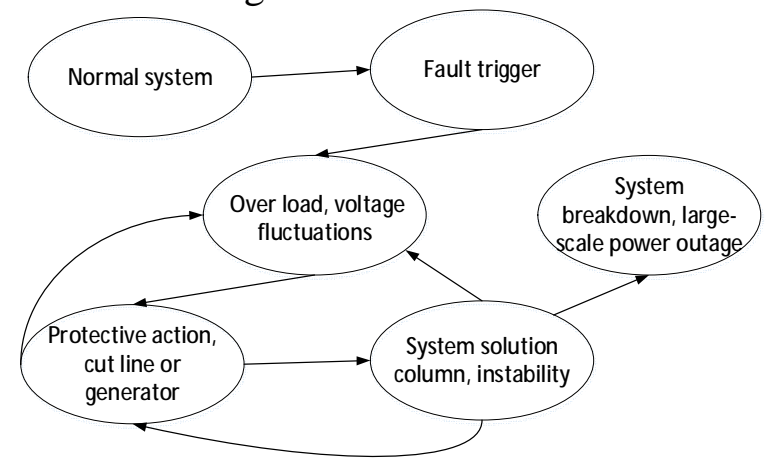

Figure 4. General development process of cascading failures

\section{Low Voltage Risk.}

The low voltage risk reflects the possibility and the harm degree of the bus voltage drop in the system:

$$
R(U \mid E, L)=\sum_{i} P_{r}\left(E_{i}\right) \sum_{j}\left[P_{r}\left(U_{i j} \mid E_{i}, L\right) \cdot S\left(U_{i j}\right)\right]
$$

Where $P_{r}\left(E_{i}\right)$ can be calculated according to the outage rate of the units under natural disasters; In the case of knowing fail units, the current situation of the system is fixed, so $P_{r}\left(U_{i j} \mid E_{i}, L\right)$ should be 1 .

\section{Unit Instability Risk.}

The difference of the power angle reflects the possibility and the degree of the system instability caused by the accident of the system: 


$$
R(\delta \mid E, L)=\sum_{i} P_{r}\left(E_{i}\right) \sum_{j}\left[P_{r}\left(\delta_{i j} \mid E_{i}, L\right) \cdot S\left(\delta_{i j}\right)\right]
$$

Similarly, $P_{r}\left(\delta_{i j} \mid E_{i}, L\right)=1$.

\section{Frequency Risk.}

Frequency is an important index to measure the stability of the system:

$$
R(f \mid E, L)=\sum_{i} P_{r}\left(E_{i}\right) \sum_{j}\left[P_{r}\left(f \mid E_{i}, L\right) \cdot S(f)\right]
$$

Similarly, $P_{r}\left(f \mid E_{i}, L\right)=1$.

\section{Cascading Failure Risk.}

Cascading failure risk index is obtained by accumulating the risk value of each stage of chain reaction:

$$
R\left(F_{c} \mid E, L\right)=\sum_{i}^{K}\left[R\left(U_{i} \mid E_{i}, L\right)+R\left(\delta_{i} \mid E_{i}, L\right)+R\left(f_{i} \mid E_{i}, L\right)\right]
$$

Where $i$ is the stage of cascading failure, $K$ is the series of cascading failures.

\section{Case Study}

In the future, SANHUA(including North China, East China, central China) regional power grid will build a number of UHV AC/DC transmission lines. The planning scheme includes Synchronous networking solutions and asynchronous networking solutions. This section will use the risk assessment theory to compare the two schemes.

If the Xiluodu, Xiangjiaba, Jinping area occurrs flood disaster, it will result in Xiluodu - Zhexi, Xiangjiaba - Shanghai, Jinping - Sunan 3 DC lines occur bipolar block at the same time. Huge active and reactive power will flood into the Yangtze River Delta region by Anhui - Zhejiang, Shanghai-Zhejiang, Fujian-Zhejiang exchange liaison passage, which may affect the system stability. Simulation analysis will be carried out below.

The transient process of the system is shown in Figure 5 and Figure 6:

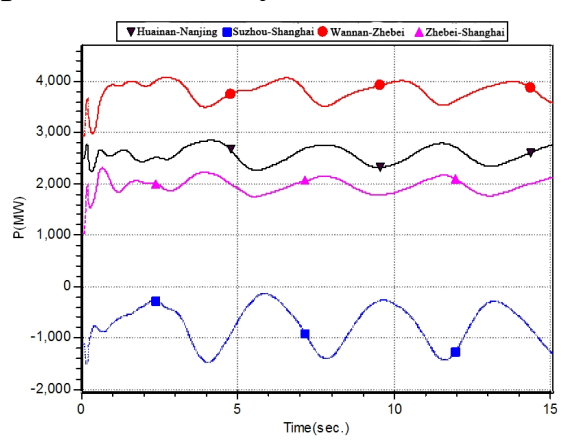

(a)Active power

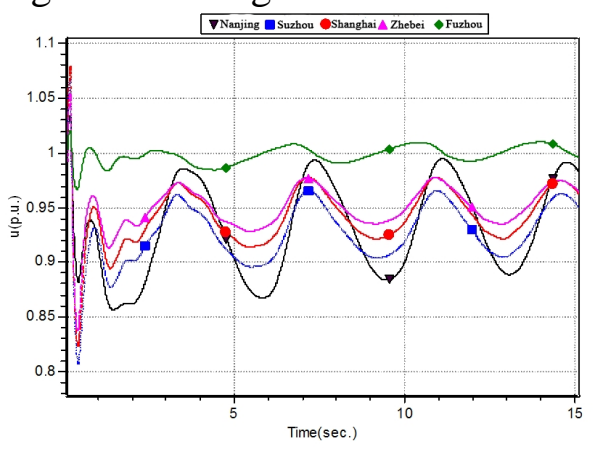

(b) Voltage

Figure 5. Synchronous networking scheme

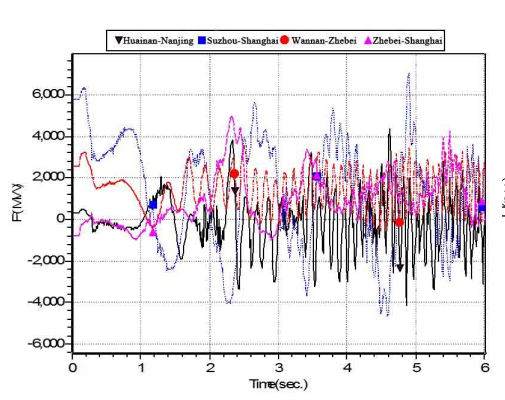

(a)Active power

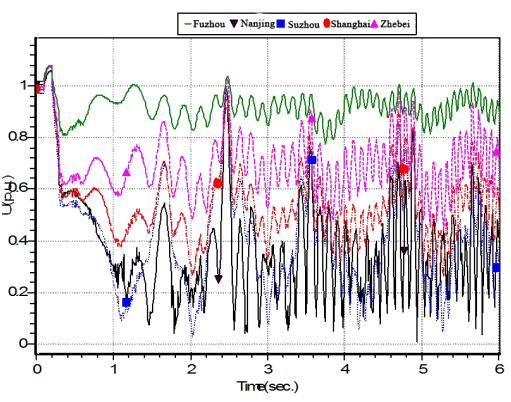

(b) Voltage 
Figure 6. Synchronous networking scheme

Take low voltage risk as an example, the risk indicators are calculated as follows:

Table 1. Results of low voltage risk indicators

\begin{tabular}{cccccc}
\hline \multirow{2}{*}{ Number } & \multirow{2}{*}{ Bus } & \multicolumn{2}{c}{ Synchronous networking scheme } & \multicolumn{2}{c}{ Asynchronous networking scheme } \\
\cline { 3 - 6 } & & Voltage (p.u.) & Risk & Voltage (p.u.) & Risk \\
\hline 1 & Guotongli & 0.71465 & 5.707 & 0.00075 & 20.015 \\
2 & Suchefang51 & 0.71835 & 5.633 & 0.00203 & 20.0406 \\
3 & Sumudu51 & 0.71868 & 5.6264 & 0.00265 & 20.053 \\
4 & Suyushan51 & 0.71902 & 5.6196 & 0.00329 & 20.0658 \\
5 & Sushibei51 & 0.73004 & 5.3992 & 0.0035 & 20.07 \\
6 & Suhuasu51 & 0.7317 & 5.366 & 0.00453 & 20.0906 \\
7 & Sukunnan51 & 0.7331 & 5.338 & 0.00468 & 20.0936 \\
8 & Guosuzhou53 & 0.73825 & 5.235 & 0.00504 & 20.1008 \\
9 & Sushuer51 & 0.75977 & 4.8046 & 0.00551 & 20.1102 \\
10 & Sushunan51 & 0.77302 & 4.5396 & 0.00075 & 20.015 \\
\hline
\end{tabular}

After calculating the unit instability risk and frequency risk, according to formula (13), the cascading failure risk is shown in Table 2 .

Table 2. Results of cascading failure risk indicators

\begin{tabular}{ccccc}
\hline UHV schemes & $R(U \mid E, L)$ & $R(\delta \mid E, L)$ & $R(f \mid E, L)$ & $R\left(F_{c} \mid E, L\right)$ \\
\hline $\begin{array}{c}\text { Synchronous } \\
\text { networking scheme } \\
\begin{array}{c}\text { Asynchronous } \\
\text { networking scheme }\end{array}\end{array}$ & 5.52 & 26.60 & 0.96 & 33.07 \\
\hline
\end{tabular}

From above results we can know that: under the above faults, the synchronization scheme can be kept stable for a period of time, and the asynchronous scheme can be lost at once, so the multiple fault ability of the synchronization scheme under this kind of fault is stronger.

Under the asynchronous scheme, East China connect with other regions through the HVDC network, if there is a lot of power vacancy, it cannot obtain the effective support. When the emergence of multiple DC line fault or large-scale power outage, due to the very large size of active power vacancy and concentrated in a small range, it will cause serious power regional imbalance, eventually lead to system instability, and loss of a large number of load; Compared with the asynchronous scheme, the synchronization scheme is connected with the DC lines to make the network synchronization, when the system is in trouble, the power flow is quickly transferred, and the active power distribution is carried out, the unbalanced active power is relatively small, and the stability is higher.

\section{Conclusions}

This paper introduced the risk assessment theory, establishes low voltage risk, unit instability risk, frequency risk and Cascading failure risk. Then the stability of the power grid cascading failures is analyzed. Simulation results show that the method is suitable for UHV AC / DC hybrid power system stability analysis. Further research will include:

(1)Combined with the actual situation of UHV power grid, we will establish a more comprehensive risk indicator system, so that the risk assessment is more comprehensive and scientific.

(2) We will combine risk assessment theory and reliability theory. We will not only analyze the reliability of the power grid, but also to consider the economic, environmental protection and other indicators, to make it more comprehensive. 


\section{Acknowledgements}

This work was financially supported by National Natural Science Foundation (51277067) and Central University Foundation (2015XS03).

\section{References}

[1] Xu M.M, Li X.Y., Bai J.L., et al. Analysis on voltage stability at commutation buses of parallel AC/DC power systems. Automation of Electric Power Systems:2009, 33(7): 6-10.

[2] XU Zheng. The analysis of dynamic behavior on AC/DC power system. Beijing: China Machine Press, 2014.

[3] Canizares C A,A lvarado F L. Point of Collapse and Continuation Methods for Large AC/DC systems, IEEE Transaction on Power System, 1993, 8(1):1-7.

[4] Liang XM, liu ZH, Wang SW, et al. Planning of UHVDC transmission system in China. Asia Pacific Region T\&D Conference,Dalian, China, 2005.

[5] CHEN Kai, DUAN Xiangying, GUO Xiaojiang. Stability Control Analysis of UHV AC/DC Hybrid Power Grid. Electric Power Construction,2016, 37(1):64-70.

[6] Ding Ming, Zhang Ruihua. Monte Carlo simulation of reliability evaluation of transmission and transmission system. Power system technology.2009, 24 (3): 9-12.

[7] Cheng Lin, Guo Yongji. Research on the adequacy and security of the transmission system. The A of the power system is dynamic, 2011,25 (20): 23-26.

[8] Hurdle EE, Bartlett LM, Andrews JD. Fault diagnostics of dynamic system operation using a fault tree based method. Reliability Engineering \& System Safety,2009,94(9): 1371-1380.

[9] Majdara A, Wakabayashi T. Component-based modeling of systems for automated fault tree generation. Reliability Engineering \& System Safety, 2009, 94(6):1076-1086.

[10] Zhang Baohui, Wang Liyong, Tan Lunnong. In the wide bright, Xie Huan. Research on the safety and reliability of power system and the risk of market environment. Power system technology, 2005, 29 (3): 44-49.

[11] Dobson I, Chen J. Thorp J S, et al. Examining criticality of blackouts in power system models with cascading events. In proc. of 35 th international conference on system sciences. Maui, Hawaii: 2012:7-10.

[12] Ming Ni, McCalley, J.D., Vittal, V" et al. Software implementation of onlinerisk-based security assessmentJEEE Trans on Power Systems, 2013,18(3):1165-1172.

[13] Wenyuan Li, J. K. Korczynski. Risk evaluation of transmission system operation modes: concepts, method and application. In Proc. of IEEE Power Engineering Society Winter Meeting, Vol 2.New York (NY, USA):2002. 1124-1129. 